\title{
Quantitative Metrics for Classifying Candidate Gun-Shot Residue Particles
}

\author{
Nicholas W. M. Ritchie ${ }^{1}$ and Dale E. Newbury ${ }^{1}$ \\ 1. NIST, Materials Measurement Science Division, Gaithersburg, MD, USA
}

The primer in a bullet produces characteristic micro-particles when a gun is discharged. These characteristic particles can come to rest on individuals who have shot a gun or were close to the discharge of a gun. The classic way to identify inorganic gun-shot residue (GSR) particles is via scanning electron microscope with energy dispersive $\mathrm{x}$-ray spectrometer (EDS). The outline of the protocol is described in ASTM standard E1588[1]. GSR particles, which are typically in the $1 \mu \mathrm{m}$ to 10 $\mu \mathrm{m}$ diameter range, are discovered using automated particle search routines. A human is required to verify that the particles found by automation are in fact 'characteristic' GSR particles where 'characteristic' usually means contains the elements $\mathrm{Pb}, \mathrm{Ba}$ and $\mathrm{Sb}$. This validation is typically implemented by manual examination of the spectrum to identify the characteristic peaks associated with each of these elements.

There are many challenges and issues with ensuring and optimizing the speed and efficiency of the automated search process. However the most important step for ensuring the credibility and integrity of our justice system is the process of assigning particles to classes. There are essentially four relevant classes of particles - 'characteristic', 'consistent', 'also associated with' and other environmental particles. 'Characteristic' particles are those particles whose composition (and to a lesser degree morphology) are "most likely associated with the discharge of a gun.[1]". By consensus, only particles containing lead, barium and antimony (potentially with a handful of elements) are uniformly deemed 'characteristic.' Consistent particles are those that "may be associated with the discharge of a gun but could also originate from other sources unrelated to a gun discharge.[1]" Not all primers produce characteristic particles. Lead free and other "non-toxic" ammunitions use primers with a different and less distinctive set of elements. Sometimes additional classes of particles may be identified for special primers or when case-specific sources are studied.

The ISO standard is vague about assignment of particles to classes. Take for example the lead-bariumantimony type. How many other elements may be present? How much lead / barium / antimony is required to say that it is present? The SWGGSR guide [2] attempts to add a little light. There is a vague discussion about major / minor / trace based on relative peak heights but this mechanism is known by the broader microanalysis community to be fundamentally flawed as nominal peak heights vary from element to element. All things considered, the standard protocols for classifying GSR-related particles are fraught with opportunity for bias and inconsistency. This is not to say that the vast majority of GSR result are not credible and defensible, rather that, particularly in edge cases, there are opportunities for unintended bias or simply the appearance of bias.

We'd like to propose a better way that is truly quantitative, statistically defensible, un-biased and reproducible. Particle spectra are notoriously difficult to quantify to extract a measure of the composition of a particle. Fortunately, we don't need to know the composition of the particle to perform robust classification. Since the earliest days of $\mathrm{x}$-ray microanalysis, the ratio of the characteristic x-ray intensity measured on an unknown sample to the same x-ray's intensity on a material of known composition (a standard), known as the 'k-ratio', is a robust mechanism for extracting elemental 
information from a spectrum. Using robust linear-least squares fitting algorithms, it is possible to extract the k-ratio with an associated uncertainty for an element from an EDS spectrum. A vector can be constructed containing the k-ratios for each element. The k-ratio vector can be normalized to mitigate mostly uninformative scale changes in the k-ratios which result from differences in particle size and geometry. The k-ratio constructed in this manner is independent of many instrumental sources of variation. So long as the spectra are collected at the same beam energy (and to a lesser extent other instrumental parameters like take-off angle), the identical particles studied on different instruments would produce statistically similar k-ratios. It is worth emphasizing that the k-ratio would also have associated uncertainty metrics. This will prove invaluable for defining what it means for an element to be present. Currie[3] discuss what it means to detect a trace quantity at various levels of utility. These rules aren't set in stone but could be used as a starting point for the GSR community to determine a quantitative, statistically defensible definition for the presence of an element.

The k-ratio vector can then be used as the input to a classification engine - a series of explicit rules that assign vectors (and thus also the associated particles) by direction into classes. There are many ways to implement a classification engine. Some classification engines assign each vector/particle into a single class. Other classification engine provide a degree-of-membership metric which quantifies the degree to which the particle fits into each class based on the evidence. Both types have strengths and both types produce robust, consistent, un-biased assignments (relative or absolute) of particles into classes.

Classification engines would have to be developed based on data collected in hundreds of gun-shot residue sampling events.

There are many ways bias and variability can be introduced into the current protocols for the analysis of GSR data. The vagueness of the definition of the various classes is one. Another is the EDS spectrum acquisition duration. The more counts in a spectrum, the more visible trace elements become. More patient analysts will see more elements than less patient ones. Another involves qualitative comparison of particles from test-firings or other case specific data with sampled particles. Decisions about which particles are selected for the comparison and how similar the particle spectra must be can bias the process. Quantitative and statistically defensible metrics can address each of these issues and others. Instrument independent standards for spectrum quality could be developed which would ensure that all labs were collecting sufficiently robust EDS spectra regardless of the relative performance of their instrument. Higher performance instruments might take less time but lower performance instruments could produce equally as reliable results with extra time. This would ensure that this small part of the implementation of justice is independent of crime laboratory.

None of what I describe here is novel within the broader domain of particle analysis. Bringing it to the GSR community would require effort to develop and validate one or more classification engines, to rewrite the GSR standard, to ensure commercial (and potentially also non-commercial) tools were available which fulfilled the standard, to train and implement the protocols in forensic laboratories. The effort would pay off with increased credibility for GSR results.

References:

[1] ASTM E1588-10 "Standard Guide for Gunshot Residue Analysis by Scanning Electron Microscopy/Energy Dispersive X-ray Spectrometry” ASTM Int'1, 2010

[2] SWGGSR, "Guide for Primer Gunshot Residue Analysis by Scanning Electron Microscopy/Energy Dispersive X-Ray Spectrometry 11-29-11”, http://www.crime-scene-investigator.net/GSRanalysisguide.pdf

[3] Currie, L. A. (1968). Limits for qualitative detection and quantitative determination. Application to radiochemistry. Analytical chemistry, 40 (3), pg 586-593. 